\title{
Mycolactone activation of Wiskott-Aldrich syndrome proteins underpins Buruli ulcer formation
}

\author{
Laure Guenin-Macé, ${ }^{1}$ Romain Veyron-Churlet, ${ }^{1}$ Maria-Isabel Thoulouze, ${ }^{2}$ \\ Guillaume Romet-Lemonne, ${ }^{3}$ Hui Hong, ${ }^{4}$ Peter F. Leadlay, ${ }^{4}$ Anne Danckaert, ${ }^{5}$ Marie-Thérèse Ruf, 6,7 \\ Serge Mostowy, ${ }^{8}$ Chiara Zurzolo, ${ }^{9}$ Philippe Bousso, ${ }^{10}$ Fabrice Chrétien, ${ }^{11,12,13}$ \\ Marie-France Carlier, ${ }^{3}$ and Caroline Demangel ${ }^{1}$
}

\begin{abstract}
${ }^{1}$ Institut Pasteur, Unité d'Immunobiologie de l'Infection, Paris, France. 2Institut Pasteur, Unité de Biologie Cellulaire des Lymphocytes, Paris, France. ${ }^{3}$ CNRS UPR 3089, Cytoskeleton Dynamics and Motility, Laboratoire d'Enzymologie et Biochimie Structurales, Gif-sur-Yvette, France. ${ }^{4}$ University of Cambridge, Department of Biochemistry, Cambridge, United Kingdom. ${ }^{5}$ nstitut Pasteur, Imagopole, Paris, France. ${ }^{6}$ Molecular Immunology, Swiss Tropical and Public Health Institute, Basel, Switzerland. 7 University of Basel, Basel, Switzerland. 8 Institut Pasteur, Unité des Interactions Bactéries-Cellules, Paris, France. 9Institut Pasteur, Unité de Trafic membranaire et Pathogenèse, Paris, France. ${ }^{10}$ Institut Pasteur, Unité des Dynamiques des Réponses Immunes, Paris, France. ${ }^{11}$ Institut Pasteur, Unité d'Histopathologie Humaine et modèles animaux, Paris, France. ${ }^{12}$ Faculté de Médecine, Université Versailles Saint Quentin en Yvelines, Versailles, France. ${ }^{13}$ Service d’Anatomie Pathologique, AP-HP, Hôpital R. Poincaré, Garches, France.
\end{abstract}

\begin{abstract}
Mycolactone is a diffusible lipid secreted by the human pathogen Mycobacterium ulcerans, which induces the formation of open skin lesions referred to as Buruli ulcers. Here, we show that mycolactone operates by hijacking the Wiskott-Aldrich syndrome protein (WASP) family of actin-nucleating factors. By disrupting WASP autoinhibition, mycolactone leads to uncontrolled activation of ARP2/3-mediated assembly of actin in the cytoplasm. In epithelial cells, mycolactone-induced stimulation of ARP2/3 concentrated in the perinuclear region, resulting in defective cell adhesion and directional migration. In vivo injection of mycolactone into mouse ears consistently altered the junctional organization and stratification of keratinocytes, leading to epidermal thinning, followed by rupture. This degradation process was efficiently suppressed by coadministration of the N-WASP inhibitor wiskostatin. These results elucidate the molecular basis of mycolactone activity and provide a mechanism for Buruli ulcer pathogenesis. Our findings should allow for the rationale design of competitive inhibitors of mycolactone binding to N-WASP, with anti-Buruli ulcer therapeutic potential.
\end{abstract}

\section{Introduction}

Mycolactone is an original polyketide metabolite synthesized by Mycobacterium ulcerans, the causative agent of Buruli ulcer (1). The life cycle of M. ulcerans is not fully understood, but there is evidence that Buruli ulcer is transmitted via insect bites (reviewed in ref. 2). Lesions typically start as a subcutaneous nodule, edema, or plaque that enlarges over time. The epidermis eventually breaks down to reveal a characteristic wound bordered by necrotic tissues (3). Mycolactone is essential for bacterial virulence and, in intradermally injected animal models, is sufficient to induce Buruli ulcer-like lesions that are marked by extensive necrosis and lack of inflammation (4). In vitro, mycolactone diffuses passively into the cytoplasm of mammalian cells to induce apoptotic cell death, although with highly variable efficacy (5). Anchorage-dependent cells are the most susceptible to mycolactone toxicity, which proceeds through cytoskeletal rearrangements and detachment (6-8). Intriguingly, at noncytotoxic doses, mycolactone also alters key functions of immune cells, such as cell trafficking and TLR-induced cytokine production (9-15). How mycolactone mediates these effects has thus far remained unknown.

Authorship note: Laure Guenin-Macé and Romain Veyron-Churlet contributed equally to this work

Conflict of interest: The authors have declared that no conflict of interest exists. Citation for this article: J Clin Invest. 2013;123(4):1501-1512. doi:10.1172/JCI66576
Given the pivotal role played by the cytoskeleton in controlling many biological processes, we reasoned that a common denominator of the activities described above could be alterations in actin dynamics. Our investigations have led us to identify Wiskott-Aldrich syndrome protein (WASP) and neural WASP (N-WASP) as molecular targets of mycolactone. Along with Scar/WAVE-1-WAVE-3, WASP and N-WASP constitute a family of scaffold proteins transducing a variety of signals into dynamic remodeling of the actin cytoskeleton, via interaction of their C-terminal verprolin-cofilin-acidic (VCA) domain with the ARP2/3 actin-nucleating complex (reviewed in ref. 16). In basal conditions, WASP and N-WASP are autoinhibited by intramolecular interactions sequestering the VCA domain from ARP2/3. Binding of activated GTPases or phosphoinositide lipids to $\mathrm{N}$-terminal target sequences triggers conformational changes resulting in release of the VCA, thereby enabling binding and activation of ARP2/3 (reviewed in ref. 17). WASP expression is restricted to hematopoietic cells, with loss- or gain-of-function mutations leading to a complex syndrome of immune defects, whereas N-WASP is more widely expressed (18). These proteins play a crucial role at the cellular level in endocytosis (19), immune synapse formation, signaling, adhesion, and migration $(16,20)$. At the tissue level, they are crucial for the maintenance of skin integrity, as shown by distorted junctions of N-WASP-depleted epithelial cells $(21,22)$ and spontaneous ulcerations in mice with conditional N-WASP deletion in the skin (23).

Using a combination of biochemical assays, cellular imaging, and animal models, we here revealed that mycolactone mimics 
A
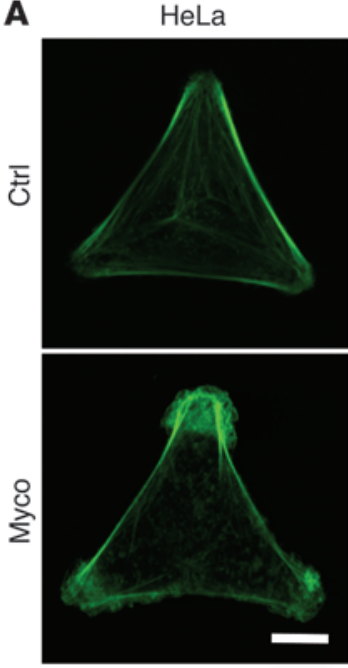

Jurkat

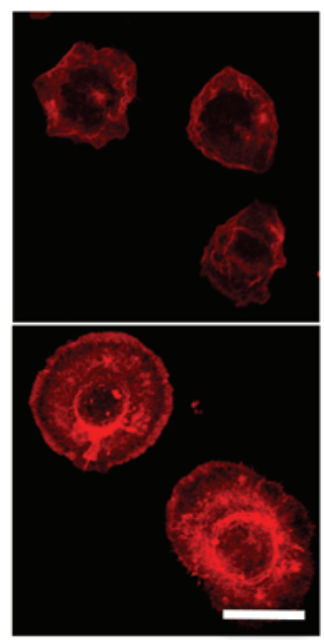

B BR CRIB

WASP 224 dkkrsg---kkkiskadigapsgfkhvshvgwdpqngfdvnnldpdlrslfsragise 278 N-WASP 186 kekkkgkakkkrltkadigtpsnfqhighvgwdpntgfdlnnldpelknlfdmcgise 243

PAK 58 sgtekg-skkkekerpeisppsdfehtihvgfdavtg-eftgmpeqwarllqtsnitk 113

WASP 279 aqltdaetskliydfiedqggleavrqemrrqe 311 N-WASP 244 aqlkdretskviydfiektggveavknelrrqa 276

PAK 114 leqk--knpqavldvlkfyd-sntvkqkylsft 140

C

WASP CR1

WASP CR2

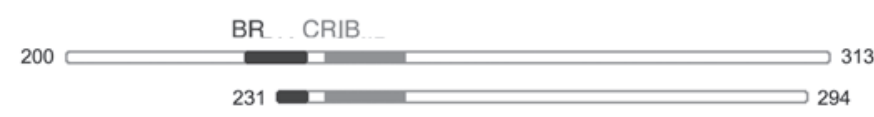

$\mathrm{N}-$ WASP CR1

$\mathrm{N}$-WASP CR2

$\mathrm{N}$-WASP CR3

N-WASP CR6

N-WASP CR7

N-WASP CR8

N-WASP CR9

N-WASP CR10

N-WASP CR11
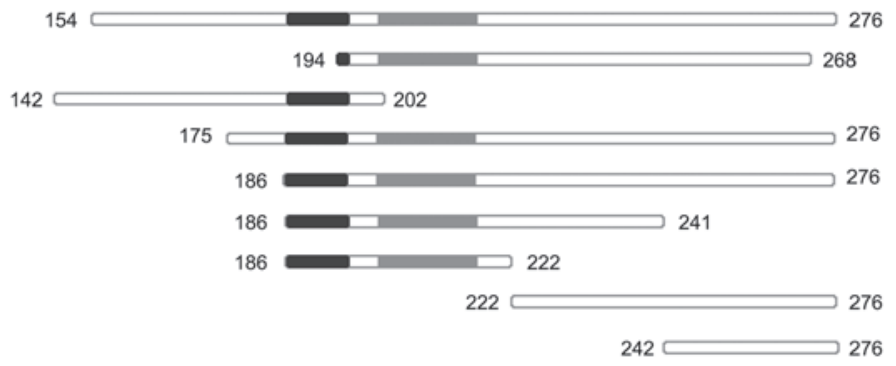

PAK CR1

52

D
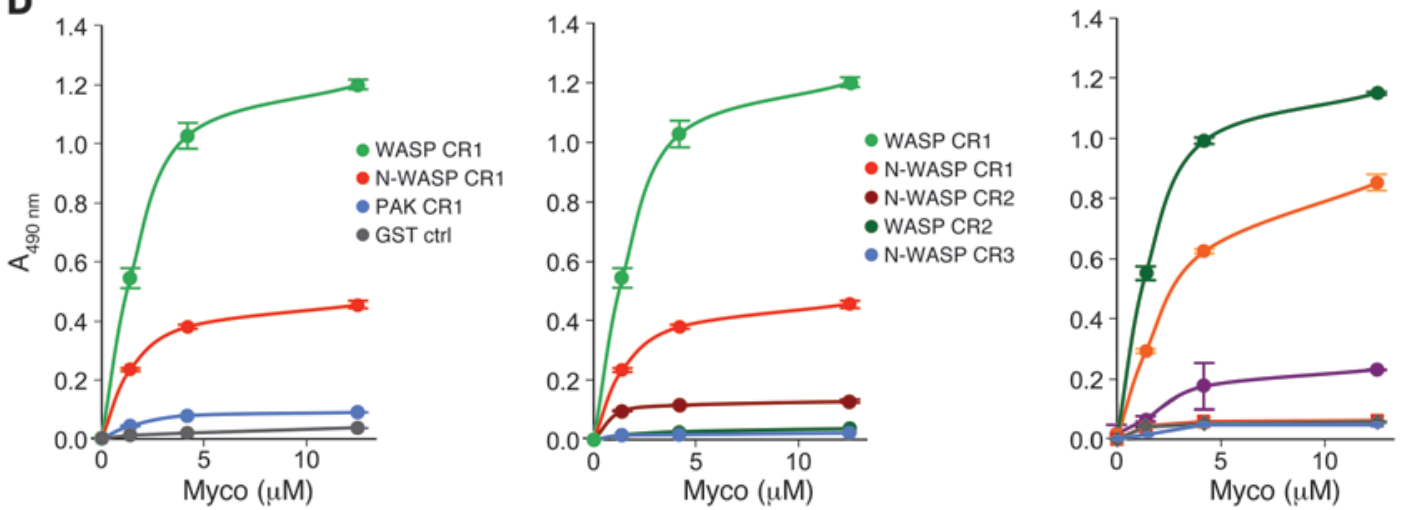

-N-WASP CR7

- N-WASP CR6

- N-WASP CR10

- N-WASP CR8

- N-WASP CR9

- N-WASP CR11 


\section{Figure 1}

Mycolactone binds to WASP/N-WASP with high affinity and specificity. (A) HeLa cells were plated on Y-shaped, fibronectin-coated micropatterns, then exposed to $20 \mathrm{nM}$ mycolactone for 30 minutes. Jurkat T cells were exposed to $20 \mathrm{nM}$ mycolactone for 5 minutes, then spun onto poly-L-lysine-coated glass slides. Actin structures were visualized by phalloidin in cells exposed to mycolactone (Myco) or solvent as control (Ctrl). Scale bars: $10 \mu \mathrm{m}$. Graphs show mean cell proportions $( \pm$ SEM) on more than 50 cells from at least 2 independent experiments. ${ }^{\star \star} P<0.01$, ${ }^{\star \star \star} P<0.001$, unpaired 2-tailed $t$ test. (B) Amino acid sequences of the GBD of WASP, N-WASP, and PAK, with boxes outlining the BR and CRIB motifs. (C) Constructs used in this study. (D) Binding of biotinylated mycolactone to these constructs in ELISA. Data

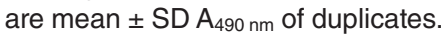

physiological signals normally delivered by Rho GTPases to hijack WASP-dependent actin polymerization. We showed that mycolactone-induced activation of N-WASP in epithelial cells and the consequent dynamic rearrangements of the actin cytoskeleton dramatically impaired the integrity of the epidermis, thus providing a molecular mechanism underpinning Buruli ulcer pathogenesis.

\section{Results}

Mycolactone binds selectively to the WASP/N-WASP regulators of actin polymerization. To analyze the effect of mycolactone on the cytoskeleton, we selected the HeLa cell line, as a model of anchorage-dependent cells of the human epithelial system, and the Jurkat cell line of human T lymphocytes, to reflect the activity of mycolactone on nonadherent, immune cells. Cells were treated with mycolactone for 5-60 minutes, then fixed and stained with fluorophore-coupled phalloidin. As shown in Figure 1A, HeLa cells exposed to mycolactone for 30 minutes produced filopodes, whereas a 5-minute treatment induced the transient formation of a large lamellipode in Jurkat cells. These observations showed that mycolactone affects the cytoskeleton in a cell-specific manner, with the filopode and lamellipode induction suggesting a modulation of actin dynamics downstream of the RAC/CDC42 GTPases. To address this possibility, we investigated a possible interaction of mycolactone with WASP, N-WASP, or p21-activated kinase (PAK), since they mediate RAC/ CDC42 signals to the actin cytoskeleton via their interactive binding (CRIB) domain (Figure 1B). Mycolactone was modified to allow covalent attachment of a biotinyl group at the end of its polyunsaturated side chain. The resulting adduct retained the biological properties of native mycolactone, as evidenced by its cytopathic and immunosuppressive activity on HeLa and Jurkat cells, respectively (Supplemental Figure 1; supplemental material available online with this article; doi:10.1172/JCI66576DS1). Biotinylated mycolactone was then used in ELISA to assess binding to the GTPasebinding domain (GBD) of WASP, N-WASP, and PAK, expressed as glutathione S-transferase (GST) fusion proteins (Figure 1C, CR1 constructs). Strikingly, mycolactone bound significantly and dosedependently to the CR1 domain of WASP and N-WASP, but not to that of PAK (Figure 1D, left).

To investigate the respective contributions of the lysine-rich basic region (BR) and the CRIB domain in CR1, we measured the reactivity of a series of constructs missing part or all of these motifs (Figure 1C). In both WASP and N-WASP, mycolactone binding was abolished when the BR was incomplete (Figure 1D, middle). However, the presence of the BR was not sufficient, as shown by the lack of reactivity of CR3 in N-WASP. Residues located upstream of the BR were not required and even diminished interaction, as evidenced by the highest reactivity of CR7 compared with CR1 and CR6 (Figure 1D, middle and right). Neither of the complementary CR7 subfragments CR8 and CR11 showed binding to mycolactone. Reactivity was partially restored with CR10, which indicates that an intact 222-276 region in N-WASP is essential. In contrast, CR9 showed no reactivity to mycolactone, further demonstrating that the BR/CRIB region is necessary but not sufficient for optimal binding. However, addition of the CRIB domain to CR10 (i.e., CR2) did not improve reactivity. Together, these results suggested that mycolactone binding to N-WASP involves a primary interaction site situated downstream of the CRIB in the 222-276 region and a secondary site in the $\mathrm{BR}$. Of all the constructs examined, CR1 in WASP and CR7 in N-WASP constituted the minimal linear domains binding mycolactone, and were therefore selected for further studies. The dissociation constants of mycolactone for CR1 and CR7 were determined by a titration assay based on tryptophan fluorescence. They were found to be comparable, in the 20- to 70-nM range (Supplemental Figure 2).

Mycolactone binding to WASPs results in CDC42-independent byperactivation. Since WASP and N-WASP use the ARP2/3 complex to stimulate actin assembly via filament branching, once the autoinhibition is relieved by binding of GTP-loaded CDC42 (CDC42GTP) $(24,25)$, we next used a pyrene fluorescence assay to examine whether mycolactone affects N-WASP activity. We found that mycolactone induced dose-dependent stimulation of the actin polymerization activity of N-WASP (Figure $2 \mathrm{~A}$ ). The maximum rate of actin assembly showed a saturation behavior, with an equilibrium dissociation constant of $170 \mathrm{nM}$ (Supplemental Figure 3). In agreement with the above observation that mycolactone bound the GBD of N-WASP, mycolactone was unable to promote ARP2/3-dependent polymerization of actin in the absence of N-WASP, nor could it stimulate a N-WASP mutant lacking the GBD (data not shown). CR7 inhibited mycolactone-induced activation of N-WASP in a dose-dependent manner, with a halfeffect of $0.25 \mu \mathrm{M}$ at $32 \mathrm{nM} \mathrm{N}$-WASP (Figure 2B), which indicates that mycolactone binds CR7 and N-WASP with similar affinity. As controls, CR2 and CR3 did not modify the mycolactone-induced activation of N-WASP (Figure 2C).

Binding of CDC42-GTP to the GBD in WASP/N-WASP triggers transition from an autoinhibited state, in which the VCA domain is sequestered by intramolecular binding to the $\mathrm{GBD}$, into an active state, where released VCA can bind ARP2/3 $(24,25)$. Since the primary interaction site of mycolactone (222-276 in N-WASP) contains most of the residues accounting for the autoinhibited fold (25), we investigated whether mycolactone affects intramolecular interactions. WASP CR1 was immobilized on beads and used to capture recombinant VCA. The resulting complex was then incubated with mycolactone, and VCA dissociation was monitored by pulldown assay. A dose-dependent release of CR1-bound VCA was induced by mycolactone (Figure 2D), which demonstrated that mycolactone disrupts WASP autoinhibition.

We then asked whether mycolactone interferes with CDC42mediated activation of WASPs, and vice versa. Mycolactone did not modify the binding of CDC42-GTP to immobilized WASP CR1 or N-WASP CR7 (data not shown). The maximal activation reached by mycolactone-bound N-WASP in actin assembly assays was more pronounced than that induced by CDC42 (Figure 2E). Moreover, the dose-dependent activation of N-WASP induced by mycolactone was independent of the presence of CDC42-GTP. 

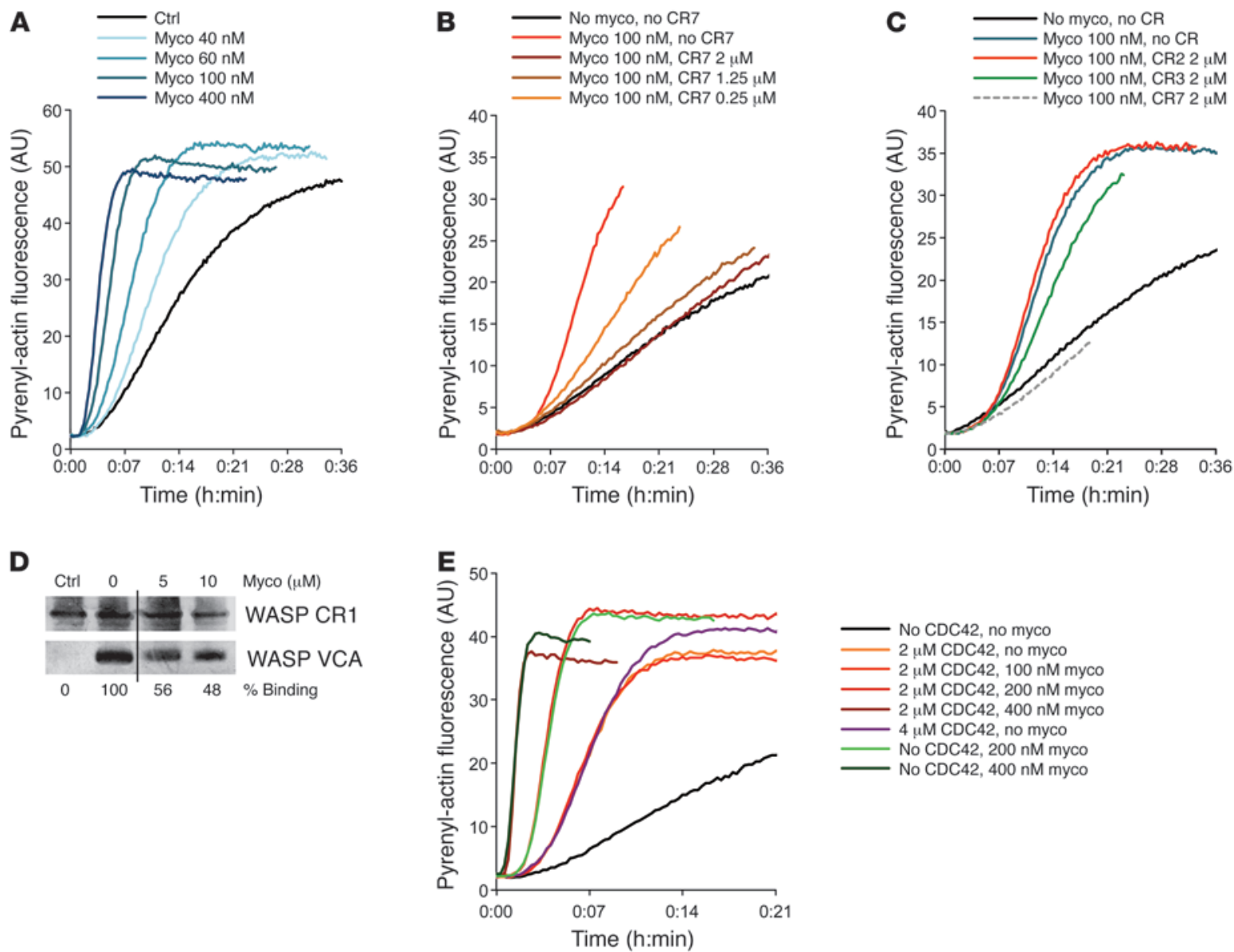

Figure 2

Mycolactone binding to N-WASP potentiates its actin polymerization activity. (A) Effect of increasing concentrations of mycolactone on N-WASPdependent polymerization of pyrenyl actin. Equivalent volumes of solvent were used as control. (B) CR7 dose-dependently inhibited mycolactoneinduced N-WASP activation. (C) Effect of CR2, CR3, and CR7 on mycolactone-induced N-WASP activation. (D) Mycolactone dose-dependently displaced CR1-bound VCA. Silver staining of WASP CR1 and VCA, after incubation of GST-fused CR1 (immobilized on glutathione-sepharose beads) with VCA in the presence of increasing amounts of mycolactone, and analysis of bead-bound products by gel electrophoresis. No VCA served as control. Lanes were run on the same gel but were noncontiguous (black line). (E) Differential activity of mycolactone and CDC42-GTP (CDC42) on N-WASP-dependent actin polymerization.

Together, these data support the view that a ternary complex can be formed in which CDC42-GTP and mycolactone bind distinct sites on WASPs. Like CDC42, mycolactone activate WASPs by releasing the VCA from the GBD. However, with a 100 -fold higher affinity (26), mycolactone is a much stronger activator of N-WASP-mediated actin assembly.

Mycolactone activates cellular WASP/N-WASP to promote actin assembly. To evaluate the physiological relevance of these findings, we then coated latex beads with mycolactone, incubated them with $\mathrm{N}$-WASP, and placed them in Jurkat cell extracts spiked with fluorescently labeled actin. A fluorescent signal readily developed at the surface of mycolactone-coated beads incubated with N-WASP, but not control beads that either had not been coated with mycolactone or had not been incubated with N-WASP (Figure $3 \mathrm{~A}$ ), which demonstrated that N-WASP activated by mycolactone could induce the formation of branched actin filaments in a cellular environment. We then took advantage of a fluorescent derivative of mycolactone to study its distribution in living cells (Supplemental Figure 4 and ref. 27). As shown in Figure 3B, myco- lactone was distributed primarily in the cytosolic compartment of treated Jurkat cells. A limited, but significant, colocalization with active WASP was observed after 1 hour, using an antibody that preferentially binds the open form of the protein (Figure 3B). Notably, spots of ARP2/3 complex were detected in the area of Bodipy-mycolactone/active WASP colocalization, which indicates that mycolactone reaches the sites of WASP-mediated ARP2/3 activation. After 4 hours, immunofluorescence detection of ARP2/3 revealed an increased level of activated complex in the perinuclear area of HeLa cells (Figure 3C). These alterations persisted for more than 16 hours and were efficiently suppressed by coincubation with the N-WASP inhibitor wiskostatin (28). They were not due to increased protein level, as expression of WASP, N-WASP, the p34 subunit of ARP2/3 (p34-ARP2/3), and actin was unchanged in mycolactone-treated cells (Figure 3D). Taken together, the data in Figure 3 indicated that mycolactone promotes perinuclear recruitment and activation of ARP $2 / 3$ by N-WASP.

Activation of $N$-WASP in epithelial cells mediates mycolactone-induced adhesion defects. Since N-WASP is a major regulator of cell adhesion 
A

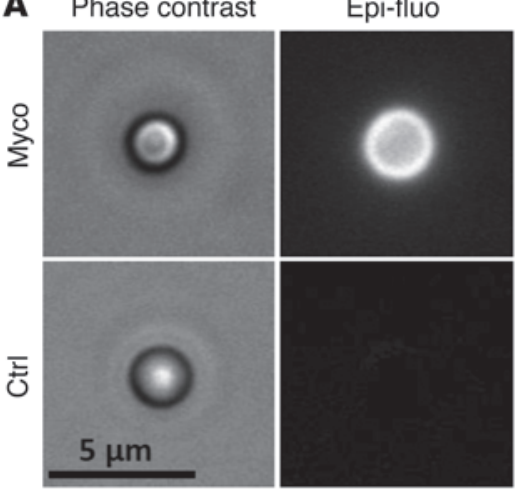

B

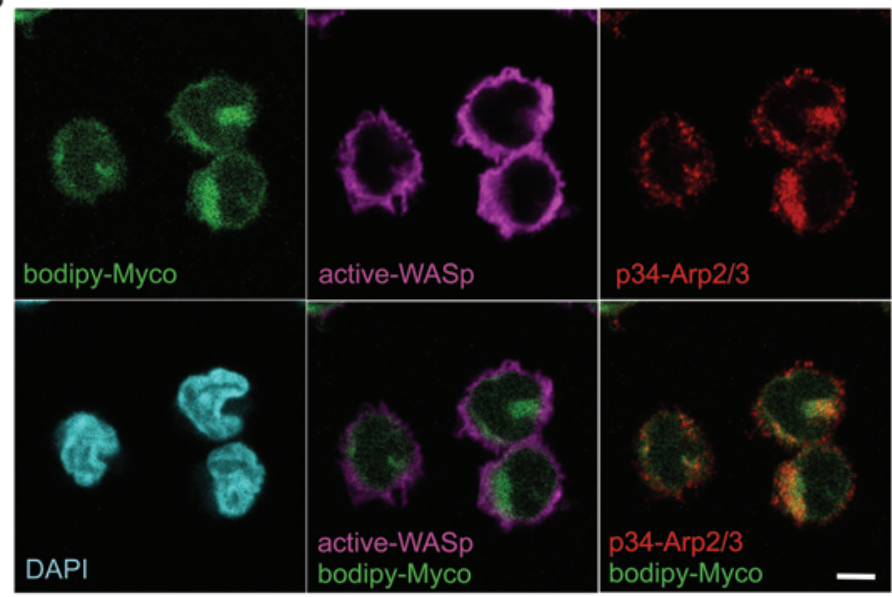

C
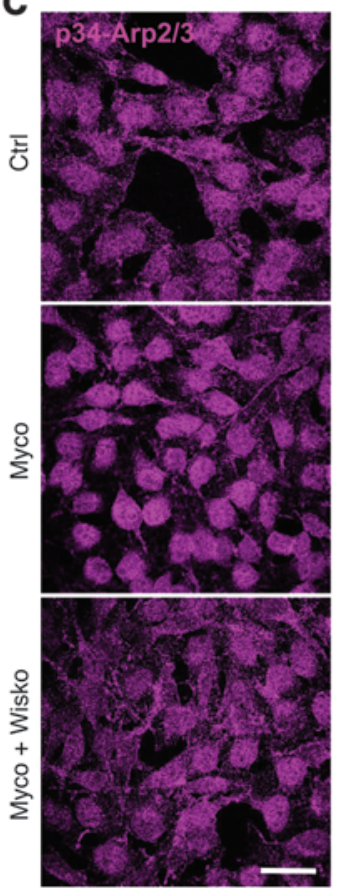

D
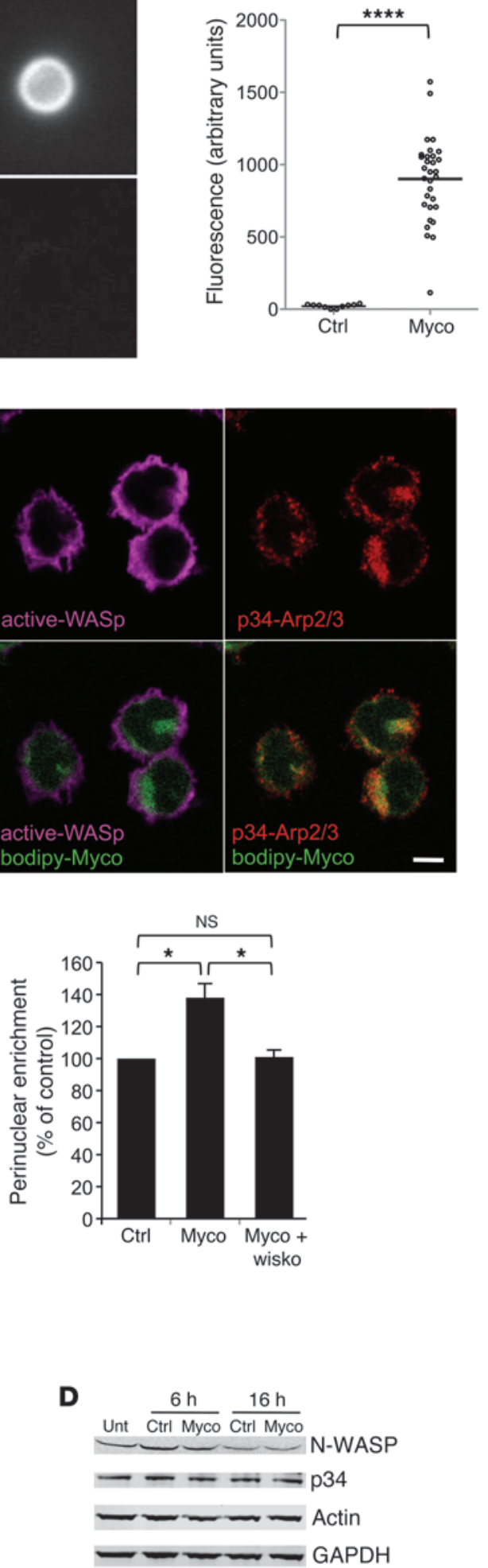

$(21,22)$, we next explored its contribution to mycolactone effects on cell-cell and cell-matrix contacts. Live imaging of HeLa cells exposed to mycolactone revealed that the early induction of spikelike protrusions was followed by lamellipodium retraction (Fig-

\section{Figure 3}

Mycolactone activates cellular WASPs. (A) Streptavidin-latex beads were coated with biotinylated mycolactone or solvent control, then incubated with N-WASP and placed in Jurkat T cell extracts supplemented with ATP, $\mathrm{MgCl}_{2}$, and Alexa Fluor 488-labeled actin monomers for 2 hours. Representative beads are shown in phase-contrast and fluorescence. Mean fluorescence signal of more than 30 beads from 3 independent experiments are compared. ${ }^{* * *} P<0.0001$, unpaired 2-tailed $t$ test, Welch corrected. (B) Jurkat cells were treated with a mycolactone fluorescent derivative (bodipy-Myco) for 1 hour, then processed for immunofluorescence with antibody binding the open form of WASP (active-WASP) and antibody recognizing $\mathrm{p} 34-\mathrm{ARP} 2 / 3$. Each image corresponds to a single confocal plane. 8 of 11 randomly picked cells had a Pearson coefficient greater than 0.5 (calculated on a $z$ stack), indicative of Bodipymycolactone and active WASP colocalization. (C) Differential ARP2/3 complex recruitment in HeLa cells treated with vehicle (control), $20 \mathrm{nM}$ mycolactone for 4 hours, or mycolactone plus $1 \mu \mathrm{M}$ wiskostatin (Wisko). Representative immunofluorescence images and integrated p34-ARP2/3 intensities $(n>50)$ in the perinuclear region (see Methods) are shown. Perinuclear enrichment was calculated as mean \pm SEM intensity and presented relative to control. ${ }^{*} P<0.05$, ANOVA with Dunn posttest. (D) Western blot analysis of N-WASP, p34-ARP2/3, and actin expression in HeLa cells exposed to $20 \mathrm{nM}$ mycolactone for 6 or 16 hours, compared with vehicle-treated control cells and untreated cells (Unt). GAPDH served as an internal control. Scale bars: $5 \mu \mathrm{m}$ (A and $\mathbf{B}) ; 25 \mu \mathrm{m}$ (C).

ure 4A). If exposure to mycolactone was prolonged over 24 hours, this process culminated in cell rounding and detachment (Figure 4, A and B). Induction of cell apoptosis, as measured by caspase activation (early stages) and membrane permeability to propidium iodide (late stages), was primarily observed in the detached fraction of mycolactone-treated cells (Figure 4C), which strongly suggested that mycolactone kills epithelial cells by anoikis. Strikingly, 4 hours of exposure to mycolactone was sufficient to induce a near-complete and persistent incapacity to adhere to plastic wells (Figure 4D). This effect was counteracted by wiskostatin or by prior transfection of the cells with WASP CR1 (Figure 4, D and E), demonstrating its association with activated N-WASP.

In HeLa cell monolayers treated with mycolactone for 16 hours, cell retraction was associated with a marked reduction in the number of intercellular contacts (Figure $5 \mathrm{~A})$. Cadherin staining showed that mycolactone suppressed both the maintenance of mature cell-cell junctions and the active formation of new interfaces via an adhesion zipper (Figure 5B). In polarized systems, such as the MDCK cell line model, mycolactone induced marked suppression of adherens and tight junctions, as shown by the decrease in the fluorescent ring-like signal of cadherins and zona occludens-1 (ZO-1; Figure 5C). Confocal imaging of basal sections revealed decreased stress fibers, increased perinuclear actin rings, and impaired membrane localization of cadherin in mycolactone-treated cells, resulting in disruption of the honeycomb-like organization and, eventually, rupture of the monolayer (Figure 5, D and E).

Mycolactone impairs direction sensing in migrating epithelial cells. The directed migration of epithelial cells requires not only activation 
A

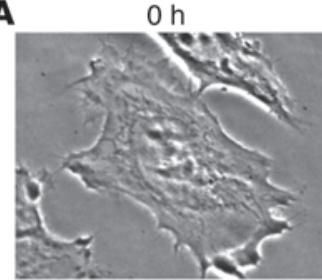

B

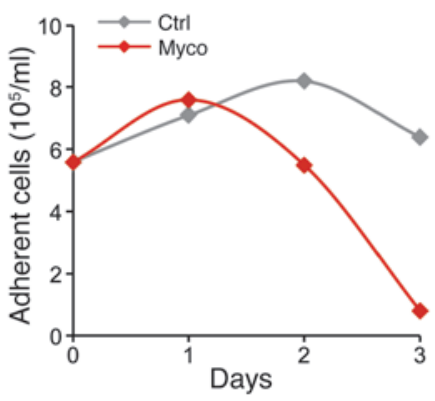

D

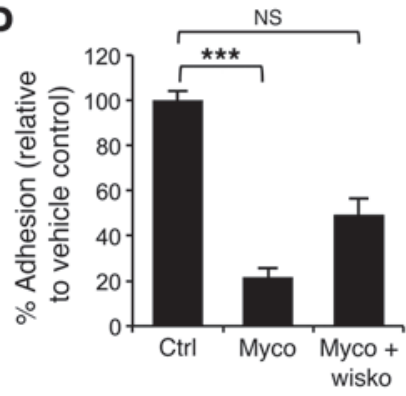

$3 \mathrm{~h}$

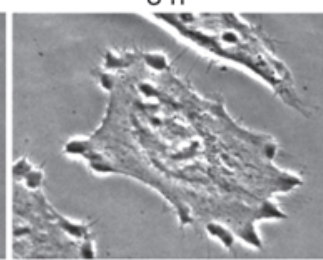

$8 \mathrm{~h}$

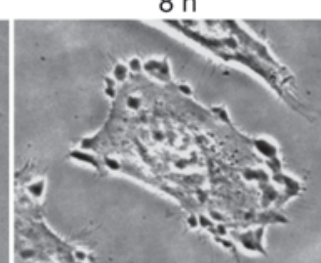

$24 \mathrm{~h}$

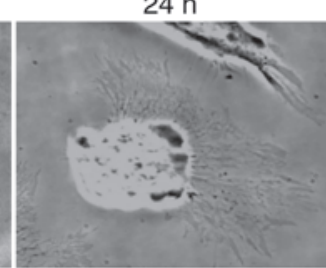

C

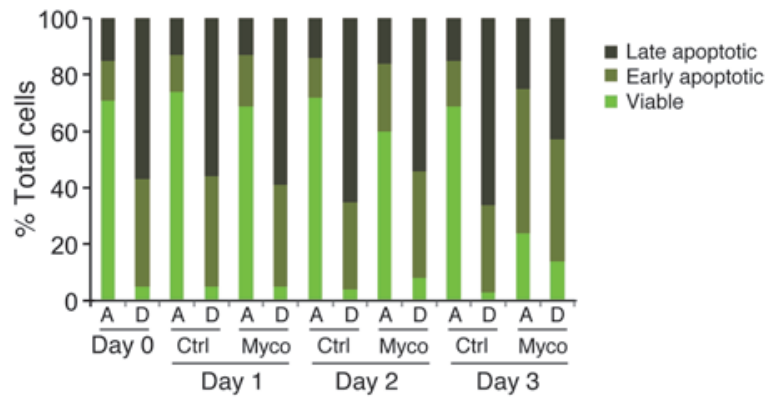

E

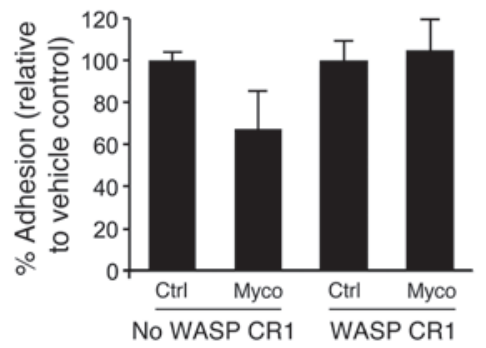

Figure 4

Effect of mycolactone on epithelial cell adhesion. (A) Phase-contrast images of a HeLa cell treated with $20 \mathrm{nM}$ mycolactone for $0,3,8$, and 24 hours. Original magnification, $\times 63$. (B) Mean number of adherent HeLa cells after treatment with $20 \mathrm{nM}$ mycolactone or vehicle control for up to 3 days. (C) Proportion of cells undergoing early and late apoptosis, in the adherent (A) and detached (D) fractions of HeLa cells treated with 20 $\mathrm{nM}$ mycolactone or solvent as control. Data are mean percentages on triplicates. (D) Adhesion of HeLa cells, as measured by calcein-AM assay, after 4 hours of treatment with vehicle control, $20 \mathrm{nM}$ mycolactone, or mycolactone in the presence of $1 \mu \mathrm{M}$ wiskostatin. ${ }^{* * \star} P<0.001$, Kruskal Wallis with Dunn post-test. (E) Adhesion of HeLa cells transfected with an expression vector encoding WASP CR1 or with an empty vector (no WASP CR1) 24 hours prior to treatment with vehicle control or $10 \mathrm{nM}$ mycolactone for 16 hours.

of ARP2/3-mediated actin assembly, but also its localization at the leading edge (29). The potential effect of mycolactone on HeLa cell displacement was therefore evaluated in a woundhealing assay. As shown in Figure 6A, mycolactone-exposed cells had an impaired capacity to close wounds. This was not due to decreased cell motility, as mycolactone-treated cells moved faster than vehicle-exposed controls (Figure 6B). However, the directionality of their movement was altered: cell trajectories were not linear and perpendicular to the wound ( $x$ axis) like those of control cells, resulting in shorter displacements (Figure 6B). Prior transfection of the cells with WASP CR1 abrogated the mycolactone-induced alterations in speed and directionality and partially restored healing capacity (Figure 6, C and D), which suggests that mycolactone binding to endogenous N-WASP is critically involved in these defects.

Hyperactivation of epithelial N-WASP underpins epidermal rupture. The human epidermis is a stratified squamous epithelium composed of proliferating basal keratinocytes attached to a basement membrane and suprabasal keratinocyte layers at various stages of differentiation (from base to top: spinosum, granulosum, lucidum, and corneum). The maintenance of cell-cell contacts and the directionality of cell migration perpendicularly to the basement membrane have been shown to be critical for the maintenance of skin integrity (reviewed in ref. 30). Since mycolactoneinduced activation of N-WASP affects both adhesion and direction sensing of epithelial cells, we investigated its effect on epidermal integrity in the mouse ear model, focusing on the events preceding tissue destruction and ulcer formation (Supplemental Figure 5). As soon as 2 days after injection, we observed progressive thinning of the external stratum granulosum and lucidum (Figure 7 , $\mathrm{A}$ and $\mathrm{B}$ ), resulting in a significant decrease in epidermis width after 54 hours (Figure 7C). In agreement with our observation on cultured cells, remodeling of the epidermal layers was associated with a dramatic loss of E-cadherin-adhesive contacts, particularly in the stratum spinosum (Figure 7D). Importantly, coadministration of wiskostatin suppressed mycolactone-induced dissociation and thinning of the epidermis (Figure 7, D and E), demonstrating the importance of N-WASP activation in this process. 
A

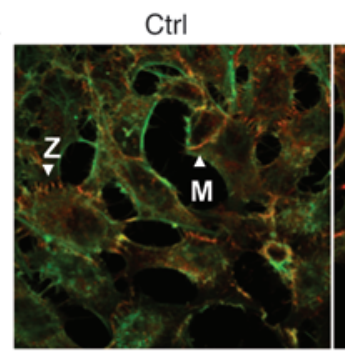

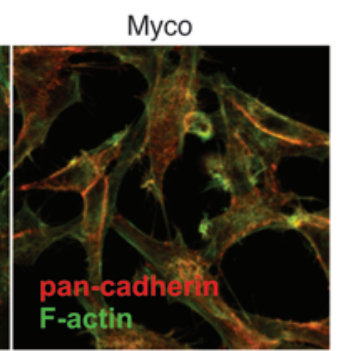

B

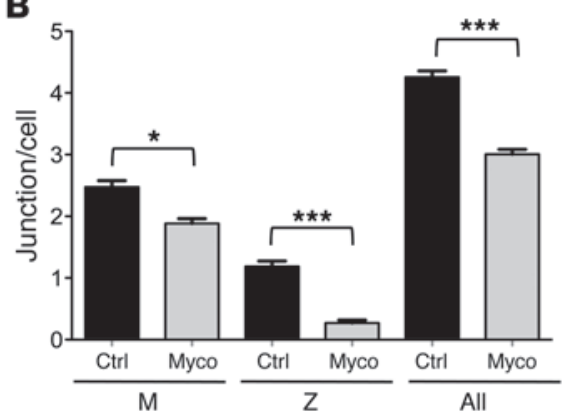

C
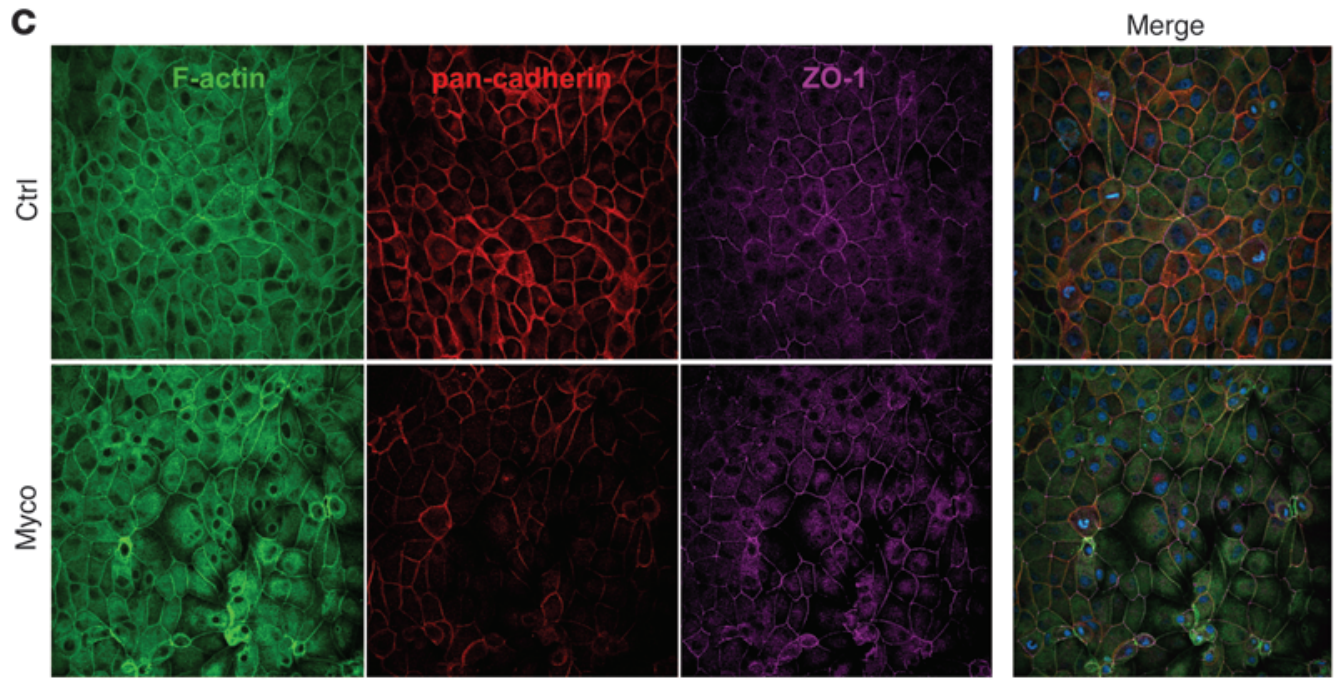

D
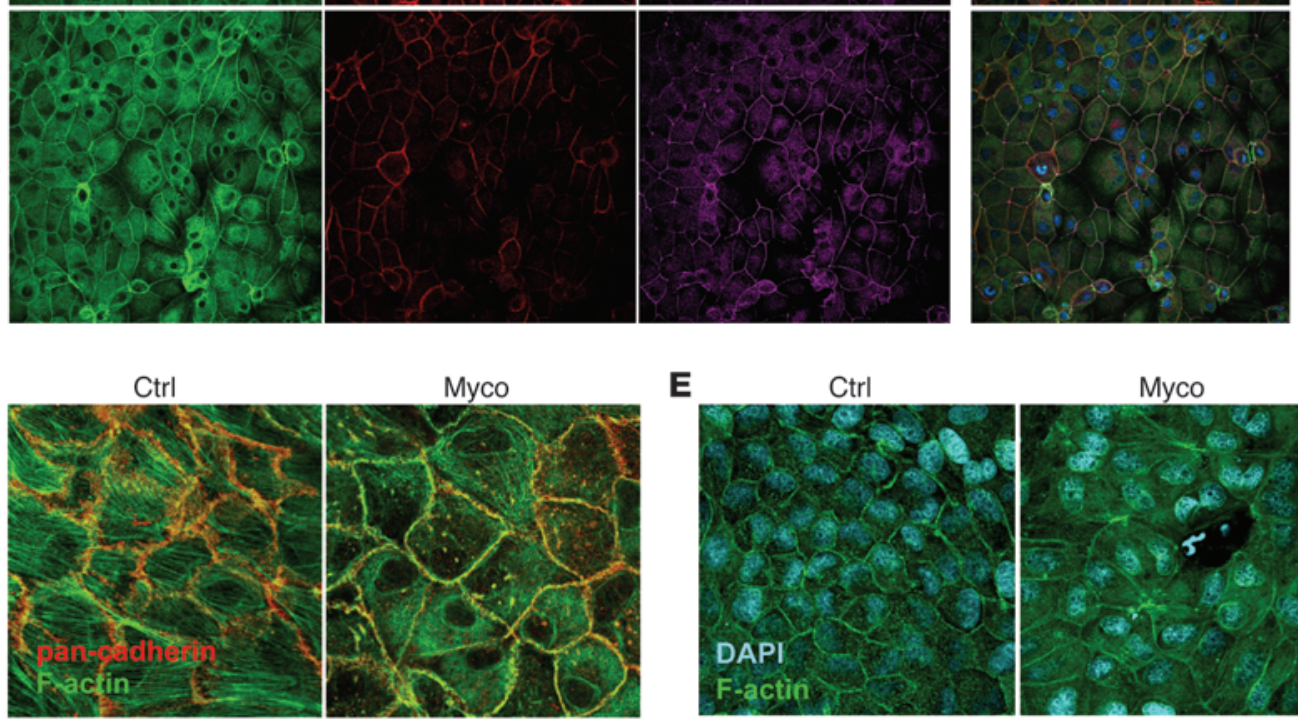

E
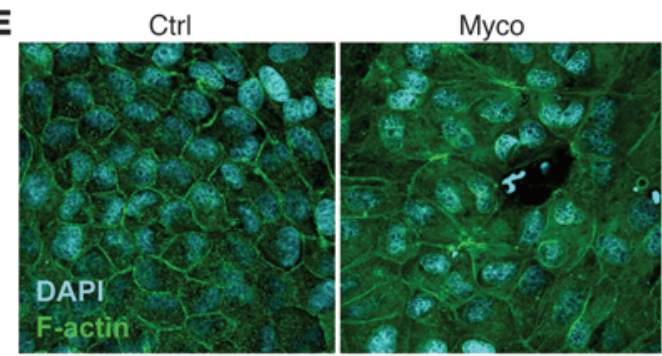

\section{Figure 5}

Effect of mycolactone on epithelial cell-cell contacts. (A) HeLa cell network after 16 hours of treatment with solvent control or $20 \mathrm{nM}$ mycolactone. Characteristic adhesion zipper (Z) and mature (M) junctions are indicated. (B) Effect of mycolactone (20 nM for 16 hours) on the number of intercellular junctions. Data are mean \pm SEM junctions per cell measured on more than 200 cells. ${ }^{*} P<0.05$, ${ }^{* * *} P<0.001$, Kruskal Wallis with Dunn post-test. (C) MDCK cell monolayer after 16 hours of treatment with solvent control or $200 \mathrm{nM}$ mycolactone. (D) Confocal image of a basal section. (E) Rupture in the epithelial layer after treatment with $50 \mathrm{nM}$ mycolactone for 16 hours. Original magnification, $\times 40$ (A and E); ×20 (C); ×63 (D).

\section{Discussion}

Here, we showed that mycolactone mimicked endogenous regulators of WASP/N-WASP to hijack ARP2/3-mediated assembly of actin filaments in host cells. Numerous bacterial effectors, such as IcsA/VirG of Shigella flexneri (31) and TccP/EspFu of the enterohemorrhagic Escherichia coli (32), target WASP/N-WASP in host cells. However, mycolactone is the first example of a nonprotein factor interfering with these actin-nucleating factors. Unlike IcsA and $\mathrm{EspFu}$, which require translocation into target cells, mycolactone gains access to WASPs by passive diffusion through the plasma membrane (8). Mycolactone bound N-WASP 100 -fold more strongly than its major regulator, CDC42 (26), leading to a much greater capacity to stimulate actin assembly in vitro. The ARP2/3 complex has previously been shown to induce a slight shift in the autoinhibitory equilibrium of WASP, facilitating CDC42-induced opening and activation (26). We found that ARP2/3 comparably potentiated mycolactone-induced release of WASP CR1-bound VCA (data not shown). This property, combined with its high affinity for WASPs, should enable mycolactone to efficiently disconnect WASPs from endogenous regulation in vivo.

Mycolactone counteracted the effects of the N-WASP inhibitor wiskostatin in cellular assays. Consistent with the fact that wisko- 
A
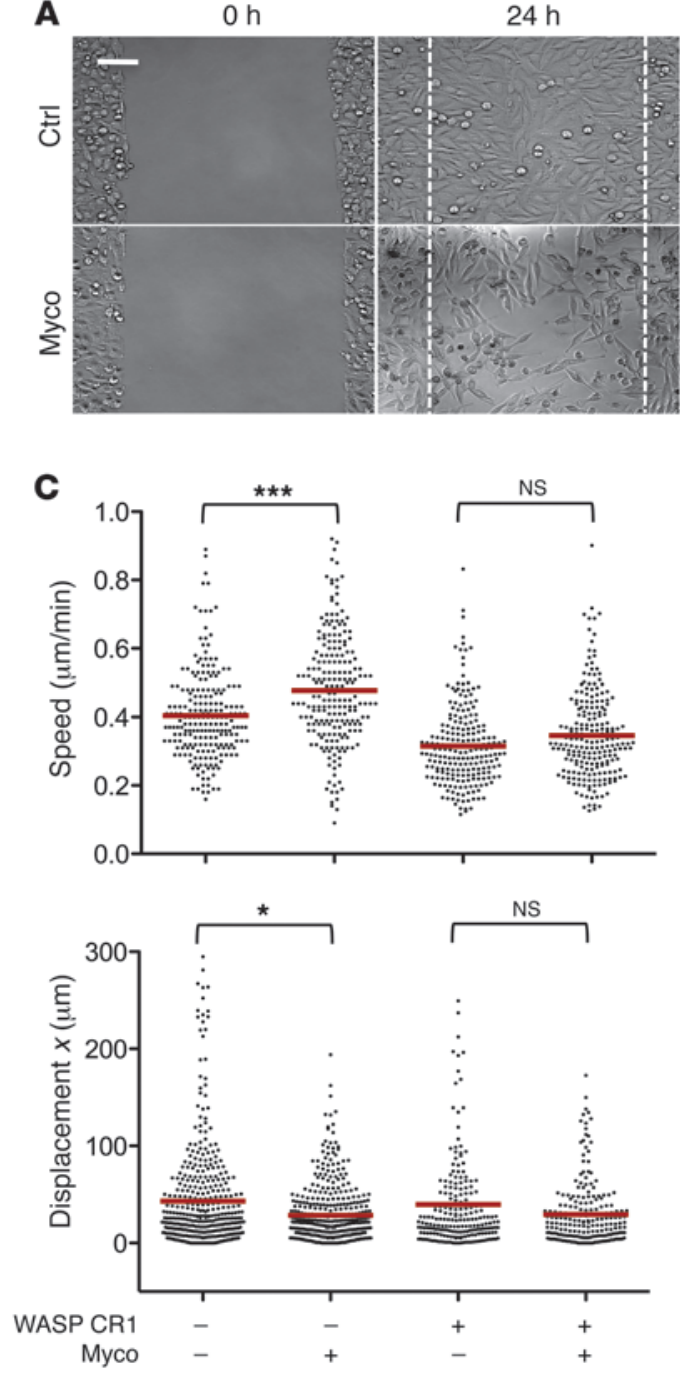

B
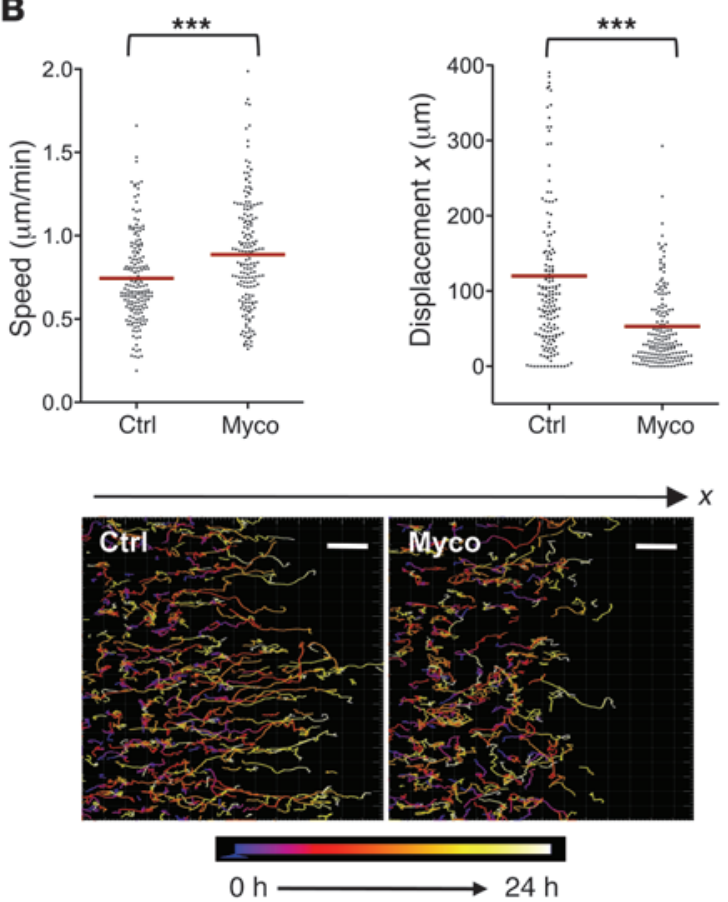

D

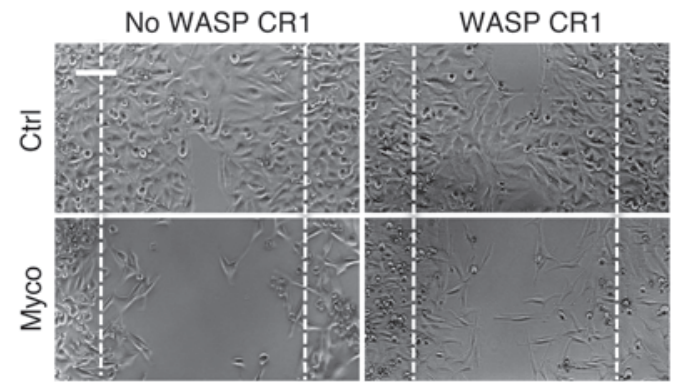

\section{Figure 6}

Mycolactone impairs directed migration of epithelial cells. (A) Representative image of the wound area after 24 hours of cell migration in the presence of $25 \mathrm{nM}$ mycolactone or methanol (control). Dashed line denotes wound edges at 0 hours. (B) Mean \pm SEM cell speed and $x$ displacement $(n>170)$, and time-dependent representation of cell trajectories. ${ }^{* \star *} P<0.001$, Mann-Whitney test. (C) Mean \pm SEM speed and $x$ displacement of more than $200 \mathrm{HeLa}$ cells transfected with an expression vector encoding WASP CR1 24 hours before assessment of cell migration in the presence or absence of $25 \mathrm{nM}$ mycolactone. Controls are cells transfected with empty vector and treated with vehicle. ${ }^{*} P<0.05$, ${ }^{* * *} P<0.001$, Kruskal Wallis with Dunn post-test. (D) Representative images of the wound area after 24 hours of cell migration. HeLa cells were transfected with an expression vector encoding WASP CR1, or an empty vector (no WASP CR1) 24 hours prior to wound formation and cell migration in the presence of $25 \mathrm{nM}$ mycolactone or vehicle control. Dashed lines denote wound edges at 0 hours. Wound-healing assays were repeated twice with similar results. Scale bars: $100 \mu \mathrm{m}(\mathbf{A}$ and $\mathbf{D}) ; 50 \mu \mathrm{m}(\mathbf{B})$.

statin inhibits N-WASP activity by stabilizing the autoinhibited conformation, we found that mycolactone relieves the intramolecular contacts that maintain WASP autoinhibition. Intriguingly, mycolactone binding to WASPs involved the BR, which is located immediately upstream of the CRIB. Phosphatidylinositol 4,5-disphosphate $\left(\mathrm{PiP}_{2}\right)$ micelles react with arginine and lysine residues clustered in this region to activate WASPs at both the allosteric level and the oligomerization level (33). NMR studies will help to determine whether mycolactone exploits this mechanism and to provide structural insight into the hyperactive conformation of the mycolactone-N-WASP complex.

In HeLa cells exposed to mycolactone, ARP2/3 was primarily detected in the perinuclear area, which suggests that mycolac- tone-induced activation of N-WASP operates preferentially in this region. Accordingly, the defects caused by mycolactone in migrating epithelial cells (e.g., increased speed of cell migration and loss of directionality) were comparable to those induced by delocalization of ARP2 expression from leading protrusions to the perinuclear area (34). The decreased capacity of mycolactone-exposed cells to extend cadherin-adhesive contacts was similar to that previously observed upon sequestration of ARP2/3 by exogenously expressing the CA region of N-WASP (35).

Our data in Figure 4 showed that mycolactone-induced killing of epithelial cells was primarily caused by N-WASP-mediated detachment and subsequent anoikis. This mechanism obviously 
A

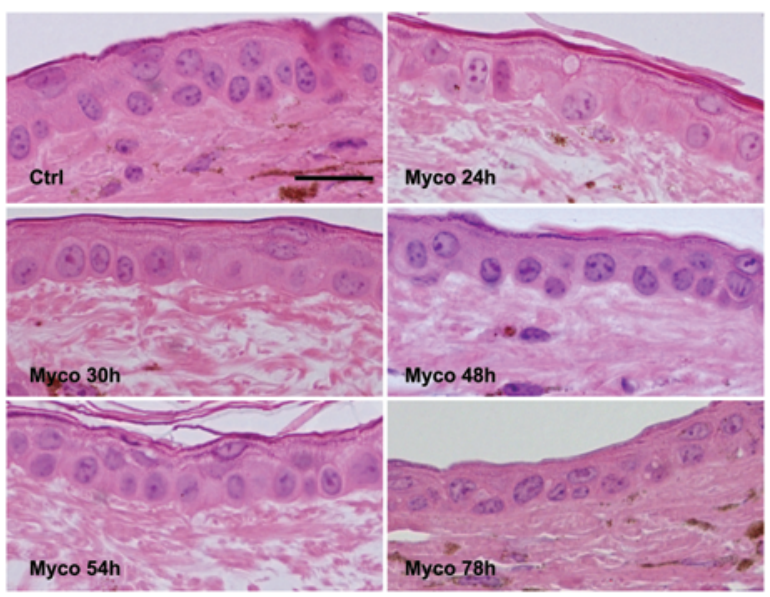

B

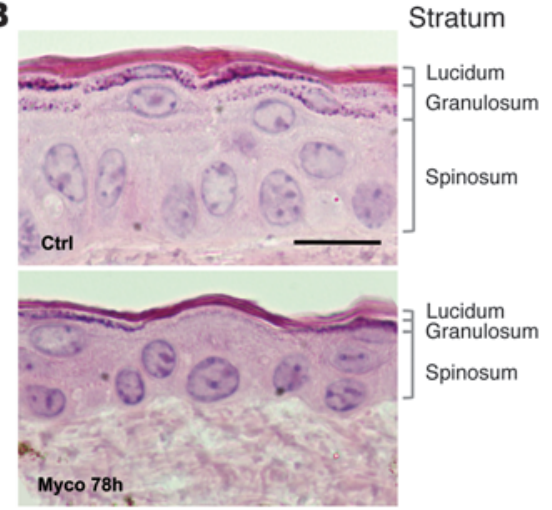

\section{C}

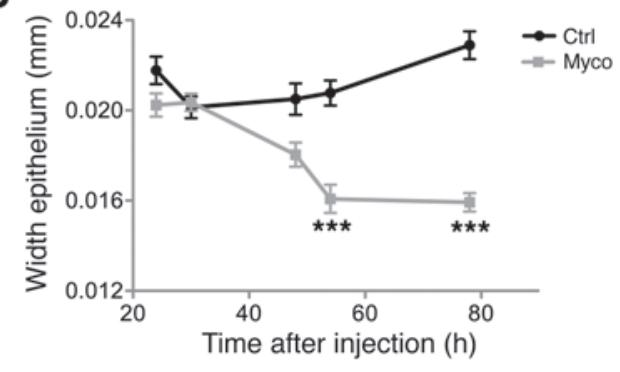

D

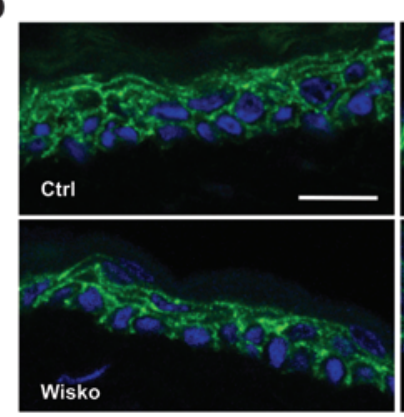

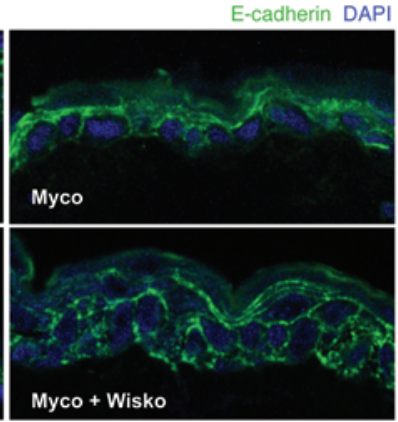

E
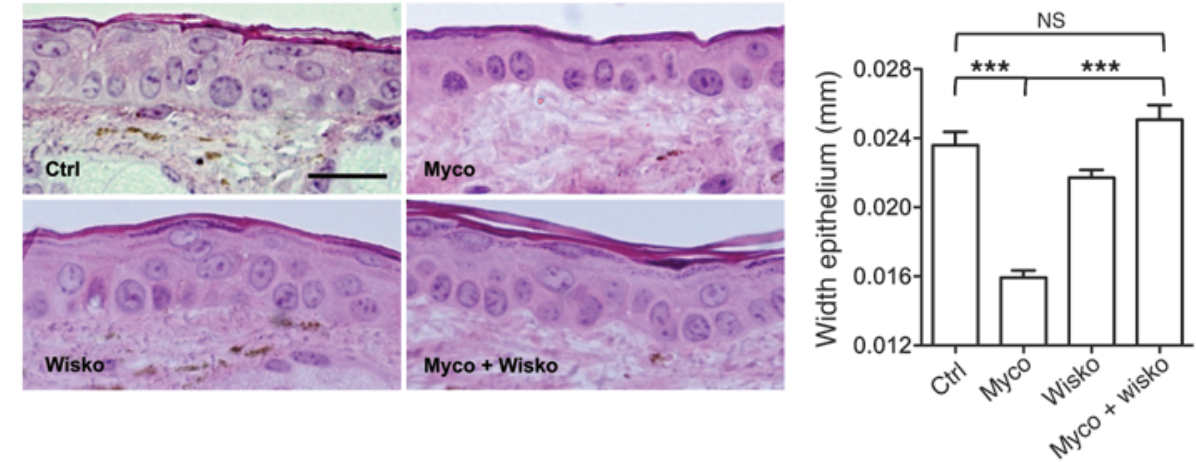

Figure 7

Remodeling of the skin epidermis after mycolactone administration. (A) H\&E-stained sections of ear skin 24, 30, 48, 54, and 78 hours after intradermal injection of vehicle control or $7 \mathrm{nmol}(5 \mu \mathrm{g})$ mycolactone. (B) Higher-magnification images of control and 78-hour mycolactone sections, showing thinning of all epidermal layers and loss of stratum granulosum. (C) Evolution of epidermal width at the site of injection of mycolactone or vehicle control. Data are mean \pm SEM from 10 measurements per mouse in 3 mice. ${ }^{* * *} P<0.001$, Kruskal Wallis with Dunn post-test. ( $\mathbf{D}$ and $\mathbf{E})$ E-cadherin staining (D) and H\&E staining and mean width (E) of the ear epidermis 78 hours after intradermal injection of $7 \mathrm{nmol}$ mycolactone, $14 \mathrm{nmol}$ wiskostatin, or both compared with vehicle control injection. Shown are representative images from 3 mice per group. Data are mean \pm SEM from 10 measurements per mouse in 3 mice. ${ }^{* * *} P<0.001$, Kruskal Wallis with Dunn post-test. Scale bars: $20 \mu \mathrm{m}(\mathbf{A}, \mathbf{D}$, and $\mathbf{E}) ; 10 \mu \mathrm{m}(\mathbf{B})$.

cannot explain mycolactone cytotoxicity in anchorage-independent cells, such as T lymphocytes (11). Activating mutations in WASP have been shown to increase apoptosis in T cells by promoting genomic instability, providing a possible mechanism for the mild cytopathic activity of mycolactone in this cell population (36). With regard to its immunomodulatory properties, recent studies indicating that WASP is an epigenetic regulator of Th1 gene expression suggest that it may be involved in mycolactone inhibition of cytokine production by $\mathrm{T}$ cells (37). Our observation that mycolactone induced lamellipode formation in T cells (Figure 1A) strongly suggests that it may also alter the $T$ cell spreading response to activation. Contrary to N-WASP, WASP is not essential and can be silenced without a major effect on viability. Study of animal models deficient for WASP expression or expressing WASP 
mutants that are unable to bind mycolactone should help to dissect the contribution of this key immune regulator to mycolactone activity in the hematopoietic cell compartment.

Currently available techniques do not allow for precise quantification of mycolactone concentration in infected tissues $(38,39)$. We chose to inject $5 \mu \mathrm{g}$ mycolactone into mouse ears, as within 2 weeks, this dose reproduces the characteristic lesions developing after months in mice infected with $M$. ulcerans (40). Consistent with our observation that mycolactone suppressed cell-cell contacts in polarized epithelia (Figure 5), our histopathological analysis of mycolactone-injected skin revealed profound alterations in E-cadherin junctions and epidermal architecture, which suggests that uncontrolled activation of N-WASP impairs epithelial differentiation in this tissue. Since mycolactone-induced thinning of the skin was suppressed by wiskostatin, we propose that hyperactivation of N-WASP (and consequent dissociation of epithelial cells) is the primary cause of epidermal destruction in Buruli ulcers.

Pathogens often modulate the host actin cytoskeleton to enhance their propagation and survival (41). M. ulcerans does not survive at the core temperature of the human body and multiplies extracellularly in disintegrated skin tissues. This environment, and the associated release of nutrients by dead cells, may constitute a favorable niche for the growth of M. ulcerans in an infected host. By targeting WASP and N-WASP, mycolactone has the potential to affect the functional biology of both immune and nonimmune cell populations. Further investigations will be required to determine whether WASP-mediated functions are altered by mycolactone in hematopoietic cells, and to what extent these alterations contribute to the immune defects of M. ulcerans-infected hosts.

Our present results suggest that modulation of mycolactoneinduced N-WASP activation in the skin might counteract the ulcerative effects of mycolactone and represent a viable strategy to treat Buruli ulcers. This hypothesis was supported by preliminary results showing that local injection of wiskostatin limited the epidermal remodeling preceding ulceration in M. ulcerans-infected mice (our unpublished observations). Resolving the structure of the mycolactone/WASP complex should identify the contact amino acids. Drugs mimicking the binding site of mycolactone in WASP may represent inhibitors of interest for treating this debilitating human disease (42).

\section{Methods}

Reagents. Mycolactone was purified from M. ulcerans 1615 (ATCC 35840) and quantified as described previously $(4,43)$. Stock solutions were prepared in methanol. Wiskostatin (Sigma-Aldrich) was in DMSO. Both reagents were diluted at least $\times 10$ in PBS before injection in animals and at least $\times 1,000$ in culture medium for cellular assays. In all cases, mycolactone- and wiskostatin-treated samples were compared with vehicle-treated controls.

WASP and N-WASP constructs. The CR1-CR7 constructs corresponding to WASP/N-WASP domains were provided by C. Egile (Institut Pasteur) or generated in our laboratory. The CR8-CR11 constructs were derived from $\mathrm{N}$-WASP CR7 for the purpose of this study. The WASP VCA construct was provided by M.K. Rosen (University of Texas Southwestern Medical Center, Dallas, Texas, USA). GST-tagged WASP/N-WASP and His ${ }_{6}$-tagged VCA constructs were produced in BL21 E. coli according to standard induction and purification procedures. Human N-WASP was expressed in Sf9 cells using the baculovirus system.

Cell cultures and viability assays. HeLa cells and Jurkat E6.1 T cells (referred to herein as Jurkat cells) were cultured in DMEM and RPMI 1640 medium (Invitrogen), respectively, supplemented with $10 \%$ heat-inactivated FCS
(Invitrogen) and $2 \mathrm{mM}$ L-glutamine (Sigma-Aldrich). Micropatterns were purchased from Cytoo, and cell plating was performed as recommended by the manufacturer. When appropriate, wiskostatin was added to HeLa cells at a final concentration of $1 \mu \mathrm{M}$ for 24 hours. This low-dose treatment had no detectable effect on cytokinesis or ATP levels (data not shown and refs. 44, 45). The induction of early/late cell apoptosis was determined by flow cytometry using the Poly-Caspases Flica assay (Immunochemistry) and Sytox-green nucleic acid stain.

Immunofluorescence microscopy. Cells were fixed for 15 minutes at room temperature (RT) with PBS plus 3\% paraformaldehyde, washed with PBS, and incubated with $\mathrm{NH}_{4} \mathrm{Cl}(50 \mathrm{mM})$ in PBS for 10 minutes at RT, prior to incubation with primary and secondary antibodies in PBS plus 5\% BSA and $0.05 \%$ saponin for 1 hour at RT. Actin was labeled with rhodamine phalloidin, cadherin with anti-pan-cadherin mouse monoclonal CH-19 (Sigma-Aldrich), ZO-1 with rabbit polyclonal 61-300 (Zymed), and N-WASP with guinea pig polyclonal antibody (provided by M.D. Welch, University of California, Berkeley, California, USA). Cell areas and junctions were measured manually on Image J pictures of actin/cadherin-stained cells. For colocalization studies, Jurkat T cells were stained with 26E6 anti-WASP monoclonal Ab (provided by J.K. Burkhardt, University of Pennsylvania, Philadelphia, Pennsylvania, USA), which is specific for the active form of the protein. WASP and Bodipymycolactone fluorescence signals were deconvoluted with Huygens professional software (Scientific Volume Imaging), and colocalization was analyzed by calculating the Pearson coefficient with the Jacop plugin of ImageJ, using Costes's automatic threshold method $(46,47)$. For ARP2/3 staining, HeLa cells were fixed with $4 \%$ paraformaldehyde in cytoskeletal stabilization buffer (10 mM PIPES, pH 6.8; 100 mM KCl; 300 mM sucrose; 2 mM EGTA; and $2 \mathrm{mM} \mathrm{MgCl}_{2}$ ) for 20 minutes, then permeabilized with $0.25 \%$ Triton X-100 in PBS for 10 minutes at RT. Cells were stained with a rabbit anti-p34-Arc/ ARPC2 polyclonal antiserum (1:400 dilution; Upstate) and anti-rabbit Cy3 (1:800 dilution), both in PBS plus 1\% BSA. Image analysis was performed with a custom-designed script developed using Acapella Image analysis software (version 2.5; Perkin Elmer). This script was based on nuclei segmentation, allowing the definition of a perinuclear ring of fixed thickness ( 6 pixels). For each nucleus $(n>50)$, the intensity of $\mathrm{p} 34-\mathrm{ARP} 2 / 3$ staining per unit of ring surface was calculated and integrated on 4 consecutive $z$ sections $(0.45 \mu \mathrm{m})$, then exported in a readable file format for statistical analysis.

Actin polymerization assays. Actin was purified from rabbit skeletal muscle (48), and the monomeric $\mathrm{G}$ form was purified by gel filtration and labeled with pyrenyl iodoacetamide or Alexa Fluor 488 succinimidyl ester (Invitrogen). The ARP2/3 complex was purified from bovine brain (31). The time course of actin polymerization was monitored spectrofluorometrically in a Safas Xenius instrument using the increase in pyrenyl actin fluorescence as a probe, with excitation and emission wavelengths of 366 and $407 \mathrm{~nm}$, respectively. Polymerization was assayed at $2.5 \mu \mathrm{m}$ G-actin (5\% pyrenyl labeled), with $28 \mathrm{~nm}$ ARP2/3 complex and $32 \mathrm{~nm} \mathrm{~N}$-WASP in the presence of mycolactone or solvent as control. The buffer contained $5 \mathrm{mM}$ Tris-Cl (pH 8), $0.2 \mathrm{mM}$ ATP, $0.1 \mathrm{mM} \mathrm{CaCl}_{2}, 0.1 \mathrm{M} \mathrm{KCl}, 1 \mathrm{mM} \mathrm{MgCl}_{2}$, and 0.2 mM EGTA. When required, CDC42 was loaded with GTP-gS by a 30 -minute incubation at RT with $1 \mathrm{mM}$ GTP-gS in $5 \mathrm{mM}$ EDTA. For actin polymerization on beads, streptavidin-latex $2-\mu \mathrm{m}$ beads were incubated with $12.5 \mu \mathrm{M}$ biotinylated mycolactone (or vehicle as control) for $15 \mathrm{~min}$ utes, rinsed, incubated with $400 \mathrm{nM} \mathrm{N}$-WASP for 30 minutes (or buffer as control), rinsed, placed into Jurkat cell homogenates spiked with $1.5 \mu \mathrm{M}$ G-actin (10\% Alexa Fluor 488) plus $1.2 \mathrm{mM}$ ATP and $2.4 \mathrm{mM} \mathrm{MgCl}_{2}$, and analyzed for fluorescence after 2 hours at RT.

Cell adhesion assays. We used the Vybrant calcein-AM-based assay of cell adhesion (Molecular Probes). Briefly, HeLa cells grown in microplate wells were treated with mycolactone or vehicle, then detached and loaded with the cytoplasmic marker calcein-AM. Labeled cells were then left to adhere 
to microplate wells for 2 hours, after which the nonadherent fraction was removed. The number of adherent cells was derived from the intensity of calcein fluorescence in each well.

Wound-healing assays. Wound-healing assays were performed with culture inserts and $\mu$-slide 8 wells from Ibidi. Briefly, HeLa cells $\left(70 \mu 1,5 \times 10^{5}\right.$ cells/ $\mathrm{ml}$ ) were seeded in culture inserts and incubated for 24 hours at $37^{\circ} \mathrm{C}$. After removal of culture inserts, cells were washed 3 times in $\times 1$ PBS and resuspended in $300 \mu \mathrm{l}$ fresh DMEM/F-12 supplemented with $10 \%$ FCS and $2 \mathrm{mM}$ L-glutamine. Cell migration was recorded for 24 hours at $37^{\circ} \mathrm{C}$ using a Nikon Eclipse Ti, capturing images at 10 -minute intervals. Cell tracking was performed with Imaris (version 6.0; Bitplane).

Histology and immunocytochemistry. C57BL/6 $\mathrm{J}$ mice were purchased from Jackson Laboratories and bred under specific pathogen-free conditions. Animals were injected intradermally in the center of the ear with the indicated reagents in a $50 \mu \mathrm{l}$ PBS volume. At the indicated time points, mice were killed, and the ears were surgically removed and divided into 2 halves. One was fixed, dehydrated with absolute ethanol, plastic resin embedded (Historesin Leica MicroSystem), and processed for H\&E staining. The other was snap frozen in isopentane cooled with liquid nitrogen before 5 - $\mu \mathrm{m}$ cryosectioning and immunostaining with rat anti-mouse E-cadherin ECCD-2 (Invitrogen) followed by secondary antibodies (Jackson ImmunoResearch). Nuclear DNA was visualized by Topro3 (Invitrogen).

Statistics. For each statistical comparison, the test used, $n$, and respective $P$ value is provided in the corresponding figure legend.

Study approval. All experiments were performed in accordance with the guidelines of the National French Veterinary Department.

\section{Acknowledgments}

The authors thank Michael K. Rosen for discussions and reagents; Philippe Sansonetti and Arnaud Echard (Institut Pasteur) for critical reading of the manuscript; Andrea Pelikan-Conchaudron (formerly at CNRS UPR 3089) and Coumaran Egile for providing WASP/N-WASP constructs; Nicolas Blanchard, Virginie Casarotto, and Anne-Caroline Chany (Université de Haute-Alsace, ENSC, Mulhouse, France) for providing fluorescently labeled mycolactone; Jost Enninga (Institut Pasteur) for help with live cell imaging experiments; Matthew D. Welch and Janis K. Burkhardt for gifts of anti-WASP/N-WASP antibodies; and Bruno Baron and Patrick England (Institut Pasteur Proteopole) for help with $K_{d}$ measurements. This work received financial support from the "Association Raoul Follereau," the ANR (project PCV 2006-2010), the European Community's Seventh Framework Programme (FP7; nos. 241500 and 241548), an ERC advanced grant (no. 229982 Forcefulactin), the "Ligue contre le Cancer," and the "Region Ile de France," which awarded R. Veyron-Churlet the postdoctoral fellowship "DIM Maladies Infectieuses, Parasitaires, et Nosocomiales Emergentes."

Received for publication August 28, 2012, and accepted in revised form January 29, 2013.

Address correspondence to: Caroline Demangel, Institut Pasteur, 25 Rue du Dr Roux, 75724 Paris, France. Phone: 33.1.40.61.30.66; Fax: 33.1.40.61.35.83; E-mail: demangel@pasteur.fr.
1. Demangel C, Stinear TP, Cole ST. Buruli ulcer: reductive evolution enhances pathogenicity of Mycobacterium ulcerans. Nat Rev Microbiol. 2009;7(1):50-60.

2. Merritt RW, et al. Ecology and transmission of Buruli ulcer disease: a systematic review. PLoS Negl Trop Dis. 2010;4(12):e911.

3. Guarner J, et al. Histopathologic features of Mycobacterium ulcerans infection. Emerg Infect Dis. 2003; 9(6):651-656.

4. George KM, et al. Mycolactone: a polyketide toxin from Mycobacterium ulcerans required for virulence. Science. 1999;283(5403):854-857.

5. Hong H, Demangel C, Pidot SJ, Leadlay PF, Stinear T. Mycolactones: immunosuppressive and cytotoxic polyketides produced by aquatic mycobacteria. Nat Prod Rep. 2008;25(3):447-454.

6. Bozzo C, et al. A Mycobacterium ulcerans toxin, mycolactone, induces apoptosis in primary human keratinocytes and in $\mathrm{HaCaT}$ cells. Microbes Infect. 2010;12(14-15):1258-1263.

7. George KM, Pascopella L, Welty DM, Small PL. A Mycobacterium ulcerans toxin, mycolactone, causes apoptosis in guinea pig ulcers and tissue culture cells. Infect Immun. 2000;68(2):877-883.

8. Snyder DS, Small PL. Uptake and cellular actions of mycolactone, a virulence determinant for Mycobacterium ulcerans. Microb Pathog. 2003;34(2):91-101.

9. Pahlevan AA, Wright DJ, Andrews C, George KM, Small PL, Foxwell BM. The inhibitory action of Mycobacterium ulcerans soluble factor on monocyte/T cell cytokine production and NF-kappa B function. J Immunol. 1999;163(7):3928-3935.

10. Coutanceau E, et al. Modulation of the host immune response by a transient intracellular stage of Mycobacterium ulcerans: the contribution of endogenous mycolactone toxin. Cell Microbiol. 2005; 7(8):1187-1196.

11. Boulkroun S, et al. Mycolactone suppresses T cell responsiveness by altering both early signaling and posttranslational events. I Immunol. 2010; 184(3):1436-1444.

12. Coutanceau E, et al. Selective suppression of dendritic cell functions by Mycobacterium ulcerans toxin mycolactone. J Exp Med. 2007;204(6):1395-1403.

13. Simmonds RE, Lali FV, Smallie T, Small PL, Foxwell BM. Mycolactone inhibits monocyte cytokine production by a posttranscriptional mechanism. J Immunol. 2009;182(4):2194-2202.

14. Guenin-Mace L, et al. Mycolactone impairs T cell homing by suppressing microRNA control of L-selectin expression. Proc Natl Acad Sci U S A. 2011; 108(31):12833-12838.

15. Torrado E, Adusumilli S, Fraga AG, Small PL, Castro AG, Pedrosa J. Mycolactone-mediated inhibition of tumor necrosis factor production by macrophages infected with Mycobacterium ulcerans has implications for the control of infection. Infect Immun. 2007;75(8):3979-3988.

16. Thrasher AJ, Burns SO. WASP: a key immunological multitasker. Nat Rev Immunol. 2010;10(3):182-192.

17. Padrick SB, Rosen MK. Physical mechanisms of signal integration by WASP family proteins. Annu Rev Biochem. 2010;79:707-735.

18. Snapper SB, et al. N-WASP deficiency reveals distinct pathways for cell surface projections and microbial actin-based motility. Nat Cell Biol. 2001; 3(10):897-904

19. Merrifield CJ, Qualmann B, Kessels MM, Almers W. Neural Wiskott Aldrich Syndrome Protein (N-WASP) and the Arp2/3 complex are recruited to sites of clathrin-mediated endocytosis in cultured fibroblasts. Eur J Cell Biol. 2004;83(1):13-18.

20. Hayward RD, Leong JM, Koronakis V, Campellone KG. Exploiting pathogenic Escherichia coli to model transmembrane receptor signalling. Nat Rev Microbiol. 2006;4(5):358-370.

21. Kovacs EM, et al. N-WASP regulates the epithelial junctional actin cytoskeleton through a noncanonical post-nucleation pathway. Nat Cell Biol. 2011;13(8):934-943.

22. Otani T, Ichii T, Aono S, Takeichi M. Cdc42 GEF Tuba regulates the junctional configuration of simple epithelial cells. J Cell Biol. 2006;175(1):135-146.

23. Lyubimova A, et al. Neural Wiskott-Aldrich syndrome protein modulates Wnt signaling and is required for hair follicle cycling in mice. J Clin
Invest. 2010;120(2):446-456.

24. Abdul-Manan N, et al. Structure of Cdc42 in complex with the GTPase-binding domain of the 'Wiskott-Aldrich syndrome' protein. Nature. 1999; 399(6734):379-383.

25. Kim AS, Kakalis LT, Abdul-Manan N, Liu GA, Rosen MK. Autoinhibition and activation mechanisms of the Wiskott-Aldrich syndrome protein. Nature. 2000;404(6774):151-158.

26. Leung DW, Rosen MK. The nucleotide switch in Cdc42 modulates coupling between the GTPasebinding and allosteric equilibria of WiskottAldrich syndrome protein. Proc Natl Acad Sci U S A. 2005;102(16):5685-5690.

27. Chany AC, et al. A diverted total synthesis of mycolactone analogues: an insight into buruli ulcer toxins. Chemistry. 2011;17(51):14413-14419.

28. Peterson JR, et al. Chemical inhibition of N-WASP by stabilization of a native autoinhibited conformation. Nat Struct Mol Biol. 2004;11(8):747-755.

29. Etienne-Manneville S. Cdc42--the centre of polarity. J Cell Sci. 2004;117(pt 8):1291-1300.

30. Etienne-Manneville S. Polarity proteins in migration and invasion. Oncogene. 2008;27(55):6970-6980.

31. Egile C, et al. Activation of the CDC42 effector $\mathrm{N}$-WASP by the Shigella flexneri IcsA protein promotes actin nucleation by Arp $2 / 3$ complex and bacterial actin-based motility. J Cell Biol. 1999; 146(6):1319-1332.

32. Sallee NA, et al. The pathogen protein $\operatorname{EspF}(\mathrm{U})$ hijacks actin polymerization using mimicry and multivalency. Nature. 2008;454(7207):1005-1008.

33. Rohatgi R, Ho HY, Kirschner MW. Mechanism of N-WASP activation by CDC42 and phosphatidylinositol 4, 5-bisphosphate. J Cell Biol. 2000; 150(6):1299-1310.

34. Liao G, Simone B, Liu G. Mis-localization of Arp2 mRNA impairs persistence of directional cell migration. Exp Cell Res. 2011;317(6):812-822.

35. Verma $S$, et al. Arp2/3 activity is necessary for efficient formation of E-cadherin adhesive contacts. J Biol Chem. 2004;279(32):34062-34070.

36. Westerberg LS, et al. Activating WASP mutations 
associated with X-linked neutropenia result in enhanced actin polymerization, altered cytoskeletal responses, and genomic instability in lymphocytes. J Exp Med. 2010;207(6):1145-1152.

37. Taylor MD, et al. Nuclear role of WASp in the pathogenesis of dysregulated TH1 immunity in human Wiskott-Aldrich syndrome. Sci Transl Med. 2010;2(37):37ra44.

38. Sarfo FS, et al. Detection of mycolactone A/B in Mycobacterium ulcerans-infected human tissue. PLoS Negl Trop Dis. 2010;4(1):e577.

39. Hong H, Coutanceau E, Leclerc M, Caleechurn L, Leadlay PF, Demangel C. Mycolactone diffuses from Mycobacterium ulcerans-infected tissues and targets mononuclear cells in peripheral blood and lymphoid organs. PLoS Negl Trop Dis. 2008;2(10):e325.

40. Coutanceau E, Legras P, Marsollier L, Reysset G, Cole
ST, Demangel C. Immunogenicity of Mycobacterium ulcerans Hsp65 and protective efficacy of a Mycobacterium leprae Hsp65-based DNA vaccine against Buruli ulcer. Microbes Infect. 2006;8(8):2075-2081.

41. Haglund CM, Welch MD. Pathogens and polymers: microbe-host interactions illuminate the cytoskeleton. J Cell Biol. 2011;195(1):7-17.

42. Johnson PD. Should antibiotics be given for Buruli ulcer? Lancet. 2010;375(9715):618-619.

43. Spangenberg T, Kishi Y. Highly sensitive, operationally simple, cost/time effective detection of the mycolactones from the human pathogen $\mathrm{Myco}-$ bacterium ulcerans. Chem Commun (Camb). 2010; 46(9):1410-1412.

44. Bompard G, Rabeharivelo G, Morin N. Inhibition of cytokinesis by wiskostatin does not rely on N-WASP/Arp2/3 complex pathway. BMC Cell Biol.
2008;9:42.

45. Guerriero CJ, Weisz OA. N-WASP inhibitor wiskostatin nonselectively perturbs membrane transport by decreasing cellular ATP levels. Am J Pbysiol Cell Physiol. 2007;292(4):C1562-C1566.

46. Bolte S, Cordelieres FP. A guided tour into subcellular colocalization analysis in light microscopy. JMicrosc. 2006;224(pt 3):213-232.

47. Costes SV, Daelemans D, Cho EH, Dobbin Z, Pavlakis G, Lockett $S$. Automatic and quantitative measurement of protein-protein colocalization in live cells. Biophys J. 2004;86(6):3993-4003.

48. Spudich JA, Watt S. The regulation of rabbit skeletal muscle contraction. I. Biochemical studies of the interaction of the tropomyosin-troponin complex with actin and the proteolytic fragments of myosin. J Biol Chem. 1971;246(15):4866-4871. 